\title{
Concomitant ipsilateral acetabular and femoral fractures - an appraisal of outcomes and complications in 34 patients
}

\author{
Umesh Kumar Meena, Mahesh Chand Bansal, Prateek Behera, Divyanshu Goyal, Rakesh Kumar
}

From the SMS Medical College, Jaipur, India

\begin{abstract}
Concomitant ipsilateral femoral and acetabular fractures are complex injuries which result from high-velocity trauma. Surgical treatment is the accepted management of such injuries. The objectives of this study were to evaluate the outcomes and study the complications in patients treated for concomitant ipsilateral acetabular and femoral fractures (type ' $B$ ' floating hip injuries).
\end{abstract}

This retrospective study was conducted at a tertiary care teaching hospital on patients operated for type B floating hip injuries, who had completed a minimum of one-year follow-up after the surgery and whose complete records were available. Those with floating hip injuries with pelvic fractures were excluded. All patients were operated on the femoral side first, followed by the acetabular side.

34 patients were included ; most of them were young males. A road traffic accident was the most common mode, with a dashboard injury being the most common mechanism of injury. No association between the type of acetabular and femoral fractures was found. The clinical (measured with Harris hip score) and radiological (Matta's method) outcomes at the latest follow-up were excellent or good in $>\mathbf{6 0} \%$ cases and had a significant association with the quality of reduction on the post-operative radiographs. Complications were seen in 12 out of the 34 patients.

Type B floating hips injuries can be managed well with acceptable short-term results by following a femur first strategy. However, patients must be informed of the possible complications and the probabilities of poorer outcomes when compared to isolated acetabular or femoral fractures.

No benefits or funds were received in support of this study. None of the authors have a conflict of interest.
Keywords : floating hip ; acetabular fracture ; pelvis fracture ; femoral fracture; floating injuries.

\section{INTRODUCTION}

Floating hip injuries have simultaneous skeletal disruptions above and below the hip joint. There must be a femoral fracture associated with either an ipsilateral pelvic fracture (Type A) or acetabular fracture (Type B) for the injury to be considered a floating hip injury $(1,2)$. Type B injuries are often considered as true floating hip injuries (1). They result from high-velocity trauma and are associated with high rates of morbidity and mortality (3). They are frequently associated with other fractures, organsystem, and neurovascular injuries. Appropriate planning of treatment is paramount for obtaining optimal results $(1,4,5)$. Life-threatening injuries of

- Umesh Kumar Meena, Assistant Professor,

- Mahesh Chand Bansal, Professor,

- Prateek Behera, Assistant Professor,

- Divyanshu Goyal, Senior Resident,

- Rakesh Kumar, Assistant Professor. Department of Orthopaedics, SMS Medical college, Jaipur, India.

Correspondence : Prateek Behera, Department of Orthopaedics, Second floor Academic Block AIIMS Bhopal, Madhya Pradesh, India, 462020.

Email : pbehera15@gmail.com

- 2021, Acta Orthopædica Belgica. 
the head, chest, and abdomen are often managed prior to performing any definitive stabilization of the skeletal injuries. Stabilization of the pelvis is essential in cases where a pelvic fracture is present as bleeding from pelvic injuries can result in lifethreatening situations.

While it is accepted that these patients require surgical intervention, there are areas where consensus is lacking. This is because these are uncommon injuries that are reported as case-reports and case series (1-18). Information on the mechanism and type of fractures, sequence of fixation, outcomes, and complications of these injuries is limited (1,3,57). There is inadequate data on the optimal timing of surgery, sequence of fixation, and complications.

While patients with type $B$ injuries can be operated first either on the pelvic side or the femoral side, at our institution the femur is operated on first followed by the acetabulum in almost all the cases. This study analyses these patients in terms of the mode and mechanism of injury, fracture types, associated injuries, parameters related to the surgical intervention, radiological and functional outcomes at their latest follow-up, and complications encountered during treatment.

\section{MATERIAL AND METHODS}

This retrospective study was conducted at a tertiary care teaching hospital after obtaining approval from the departmental review board. Data of patients treated for pelvi-acetabular trauma between January 2013 and January 2019 was retrieved from records, which were screened to select patients with Type B floating hip injuries. Patients with complete details of the parameters being studied, follow-up, and outcome data were selected; those with a concomitant pelvis and femur fracture but without any acetabular fracture were excluded. These details were collected in a pre-defined proforma (Tables I and II). Acetabular fractures were classified according to Leturnel and Judet classification $(19,20)$. Femoral fractures were classified based on the anatomical location of the fracture and the AO classification.

All patients were operated upon on a radiolucent operating table. Surgical approaches for the aceta- bulum and implants for femoral and acetabular fractures were decided by the surgeon based on the fracture type. Whether the surgery was completed in one or two anaesthetic sittings was a decision of the anaesthesiologist. Decisions were based primarily on the patients' condition after the femoral surgery and the expected duration and blood loss in the acetabular surgery.

All patients received low molecular weight heparin (LMWH) for deep vein thrombosis prophylaxis for 4 weeks and indomethacin $(25 \mathrm{mg}$ thrice a day) for heterotopic ossification prophylaxis for 6 weeks. Non-weight-bearing ambulation was permitted from the second post-operative day. However, weight-bearing was allowed after 3-12 weeks of surgery depending on fracture type, degree of comminution, and stability of fixation. The quality of reduction was assessed on the immediate postoperative radiographs using Matta's method (19). Patients were advised for follow-up at 2 weeks, three months, and every six months thereafter.

Radiographs at the latest follow-up were used for assessing radiological outcome by Matta's scoring system (19). Clinical outcome was evaluated using Harris hip score at each visit after one year. Heterotopic ossification was classified using the method described by Brooker et al. (18).

The data was analysed using SPSS version 26 (IBM Corp., Armonk, NY). The frequency was calculated for categorical variables. Mean values were calculated for continuous data. Fisher's exact test was used for evaluating clinical and radiological outcomes with the reduction quality on the postoperative radiographs. A 'p' value of $<0.05$ was considered significant.

\section{RESULTS}

565 patients of pelvic-acetabular trauma were treated during the study period. 44 patients with Type B floating hip injuries were operated on. 10 patients were excluded as the required details were unavailable (incomplete data or follow-up). 34 patients were thus included. Among these 33 were males and 1 was female. Road traffic accident (32 patients, 94.1\%) was the most common mode of trauma. Dashboard injury was the commonest 


\begin{tabular}{|c|c|}
\hline 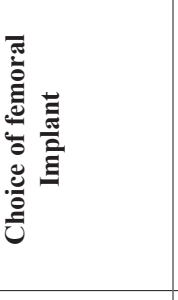 & 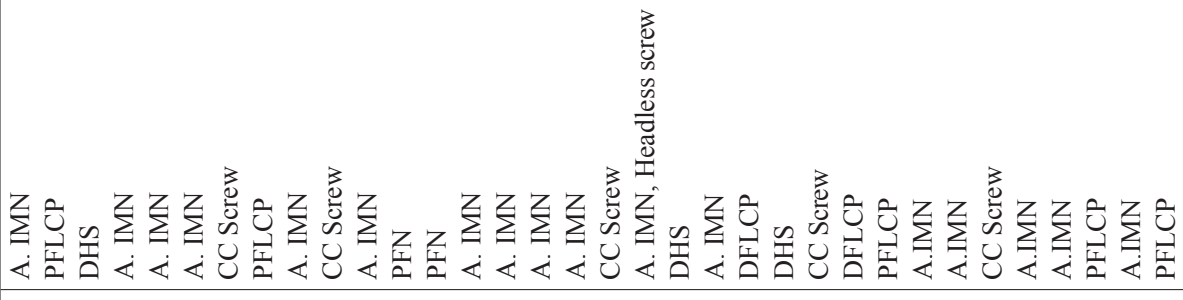 \\
\hline 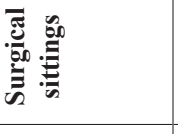 & 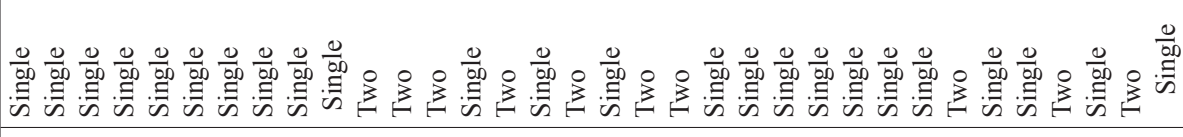 \\
\hline 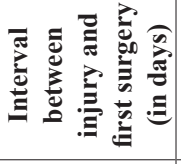 & 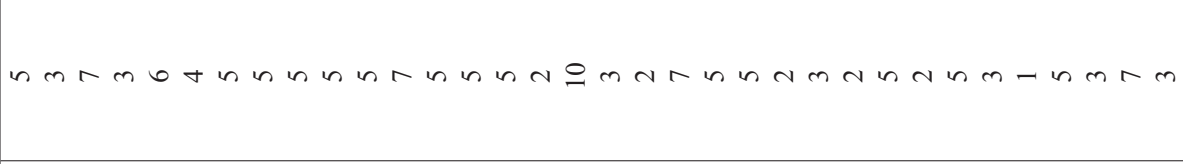 \\
\hline 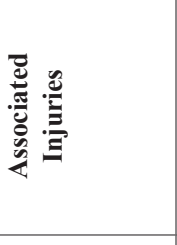 & 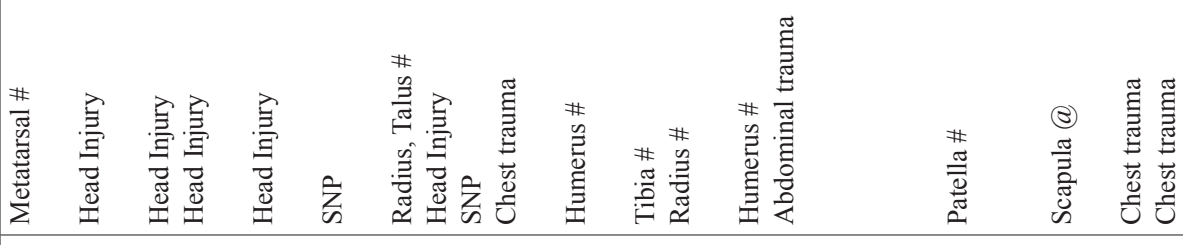 \\
\hline 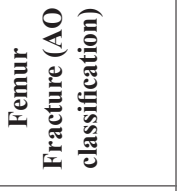 & 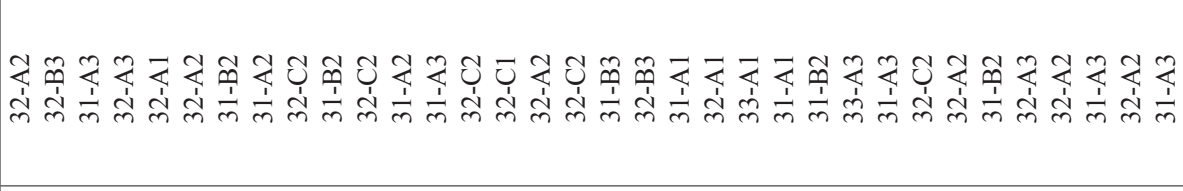 \\
\hline 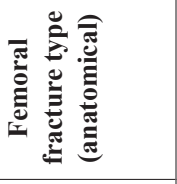 & 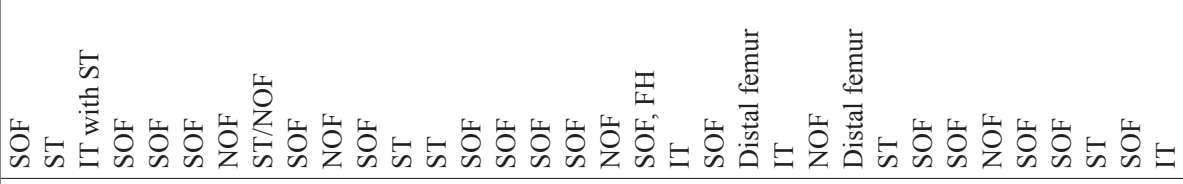 \\
\hline 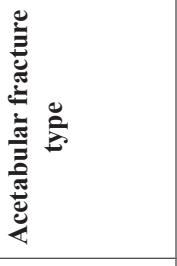 & 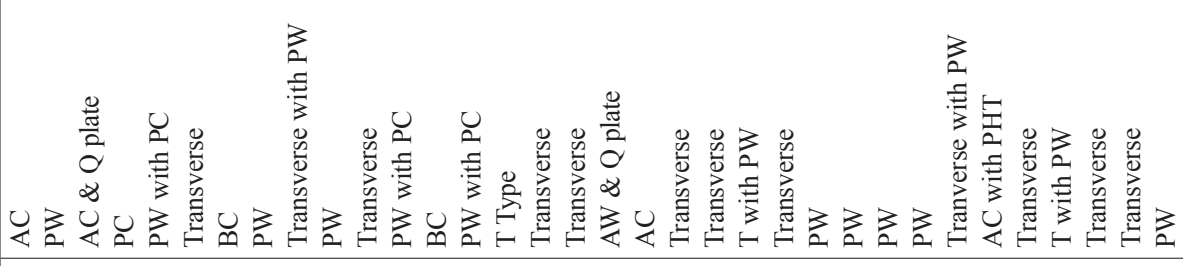 \\
\hline 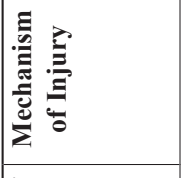 & 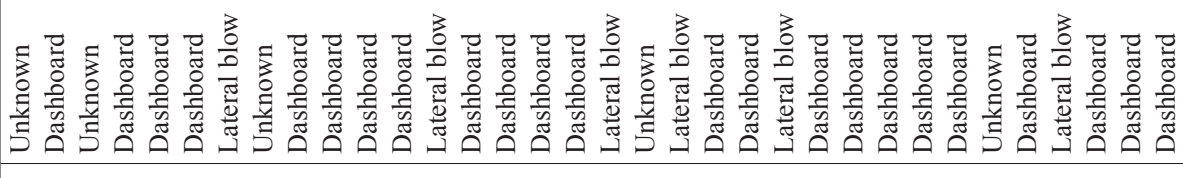 \\
\hline 递 & 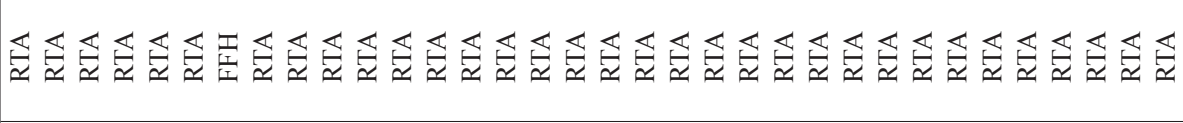 \\
\hline 离 & 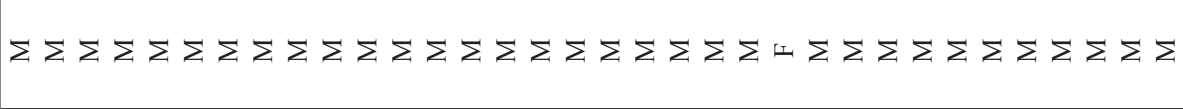 \\
\hline 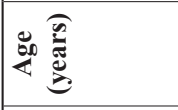 & |q \\
\hline 言 & $-N m+n$ - \\
\hline
\end{tabular}

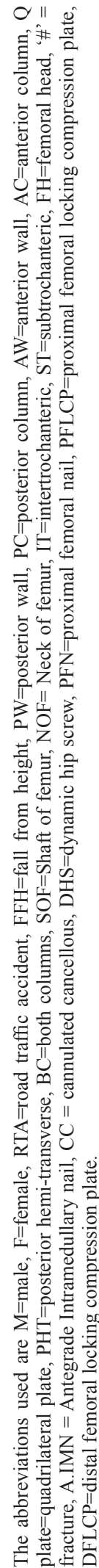




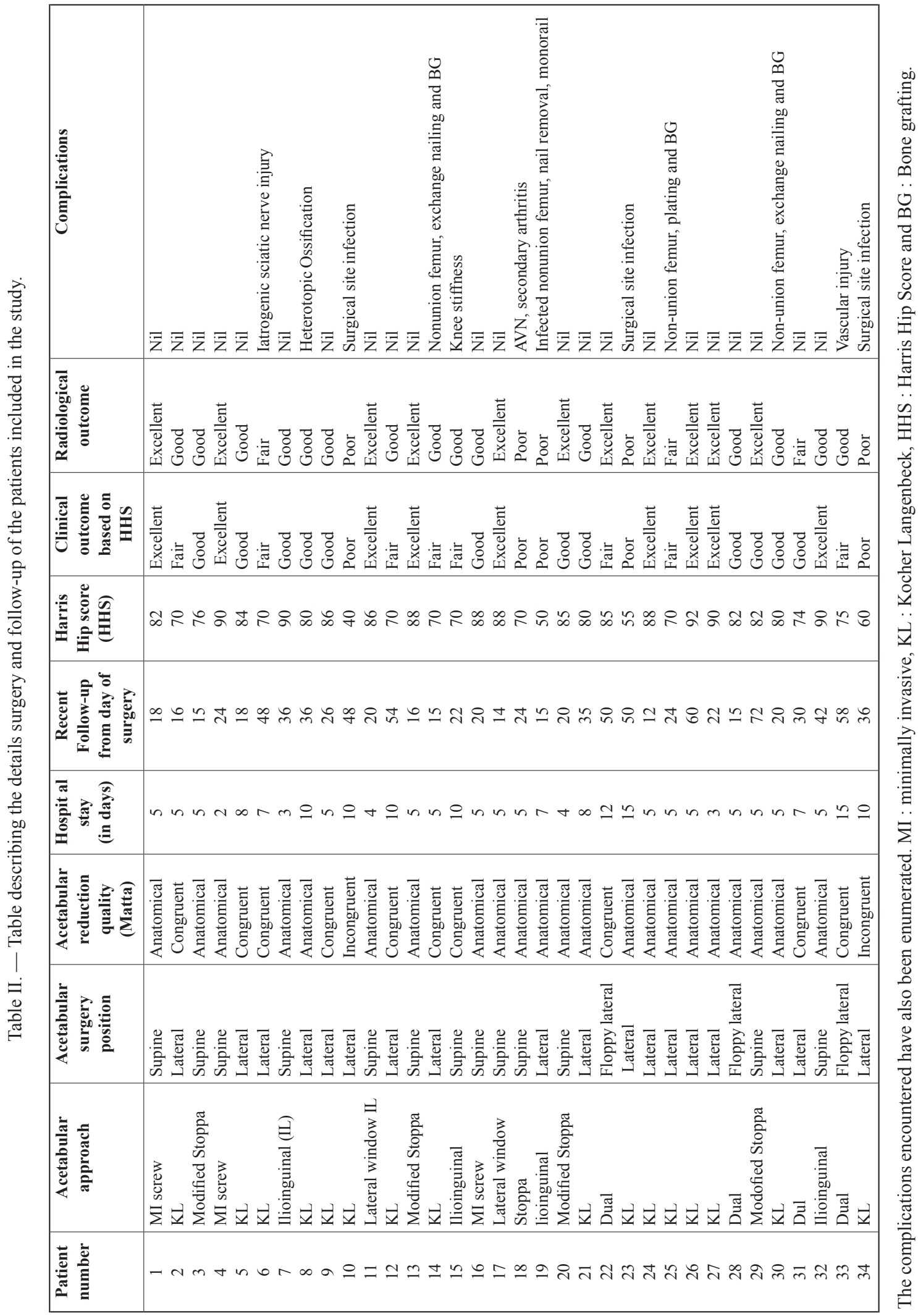




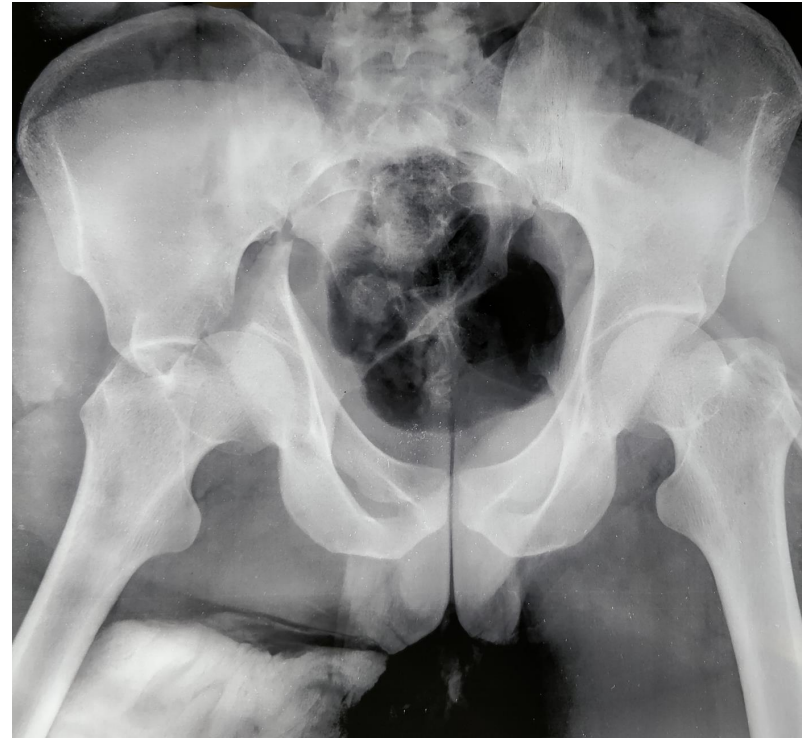

Fig. 1A. - Anteroposterior radiograph of a patient showing a transverse fracture of the right acetabulum.

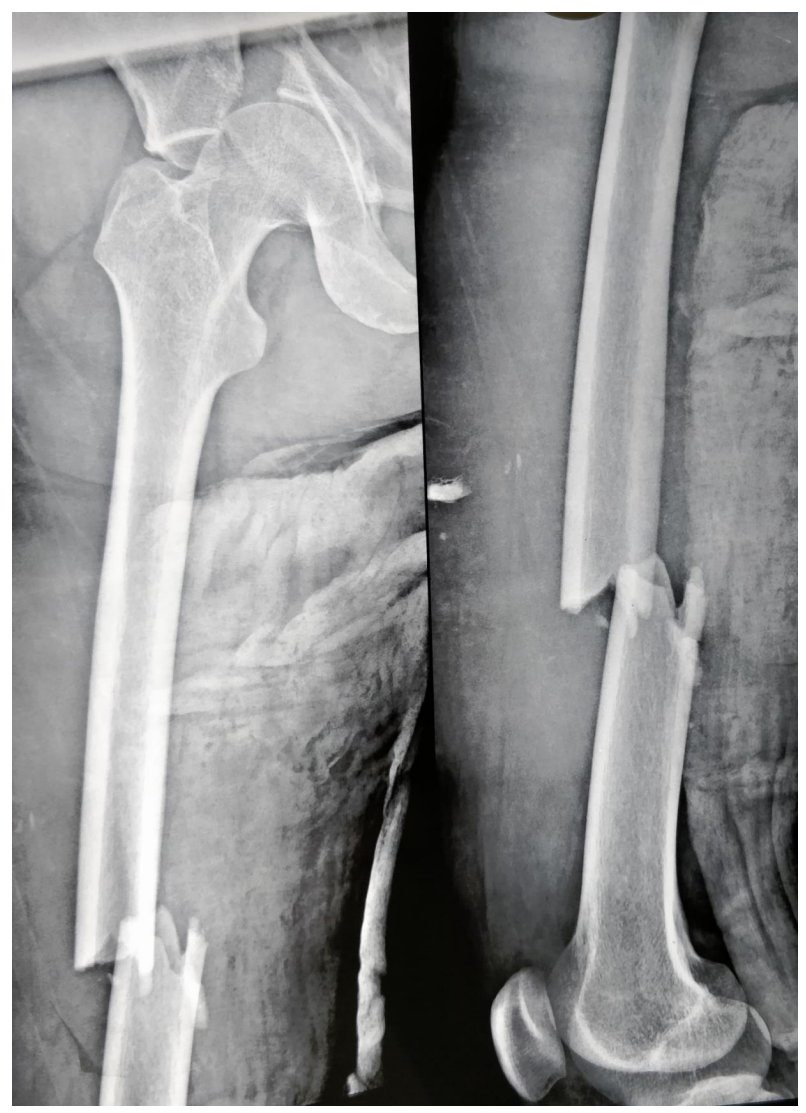

Fig. 1B. - Anteroposterior and lateral radiographs of the patient showing a concomitant ipsilateral femoral shaft fracture.

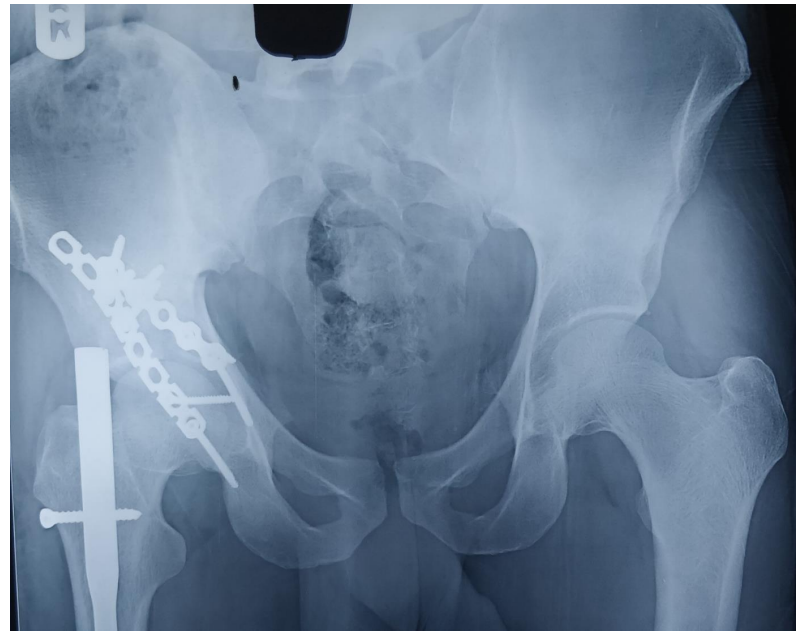

Fig. 1C. -20 months post op radiograph of the acetabular fracture showing good radiological outcome of the acetabular fracture.

mechanism of injury (23 patients, $67.6 \%$ ) with lateral blow reported in 6 patients $(17.6 \%)$. Transverse fracture (10 patients, 29.4\%) was the commonest acetabular fracture type followed by posterior wall ( $\mathrm{PW}$ ) fracture (8 patients, $23.5 \%$ ).

19 patients $(55.9 \%)$ had associated injuries. A fracture of the lower or upper limb was the most common associated injury ( 8 patients). Injury to the head, chest, and abdomen was seen in 5, 3, and 1 patient, respectively. Post-traumatic sciatic nerve palsy was present in 2 patients. The mean interval between injury and first Orthopaedic surgical intervention was 4.41 days. Closed reduction of the hip was done in all patients who presented with a dislocation at the time of admission and if the hip was found to be unstable or non-reducible, the surgery was performed on an emergency basis. $24(70.6 \%)$ cases were operated on in a single anaesthetic sitting and $10(29.4 \%)$ in two sittings after an interval of 2 to 5 days (Table I). Antegrade intramedullary nailing (IMN) was done in all 17 patients with femoral shaft fractures. Among these, 7 patients (PW, posterior column (PC), and transverse fractures) had femoral nailing and acetabular fixation in the lateral position in the same anaesthetic sitting (Figures 1A-1C). 3 patients with femoral shaft fractures who had un-displaced or minimally displaced PC or anterior column (AC) 


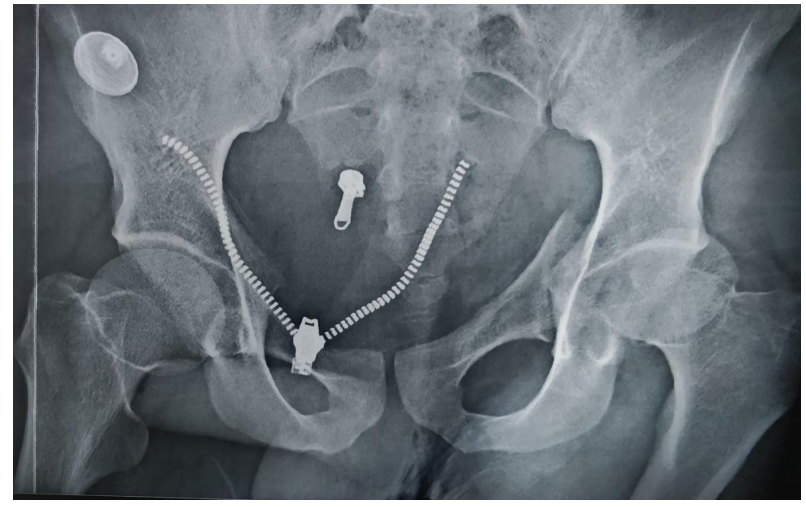

Fig. 2A. - Anteroposterior radiograph of a patient (number 19 in tables I and II) showing anterior column fracture with a femoral head fracture.

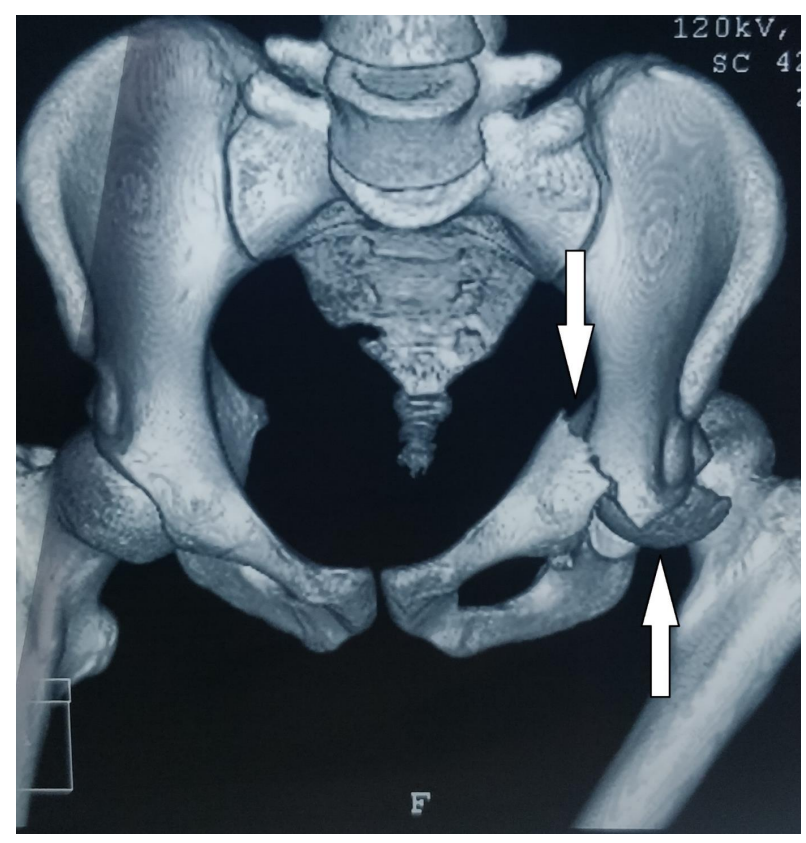

Fig. 2B. - 3D CT reconstruction showing the anterior column fracture (down pointing arrow) and femoral head fracture (up pointing arrow).

fractures had minimally invasive screw fixation of the columns after IMN. One patient (patient 19 in Tables I and II) had an AC fracture with a femoral head and shaft fracture. He also had a tibial shaft fracture. He was operated on in two sittings. In the first sitting, femoral IMN followed by femoral head fixation by safe surgical dislocation was done. Tibial IMN was done too. The AC was fixed after

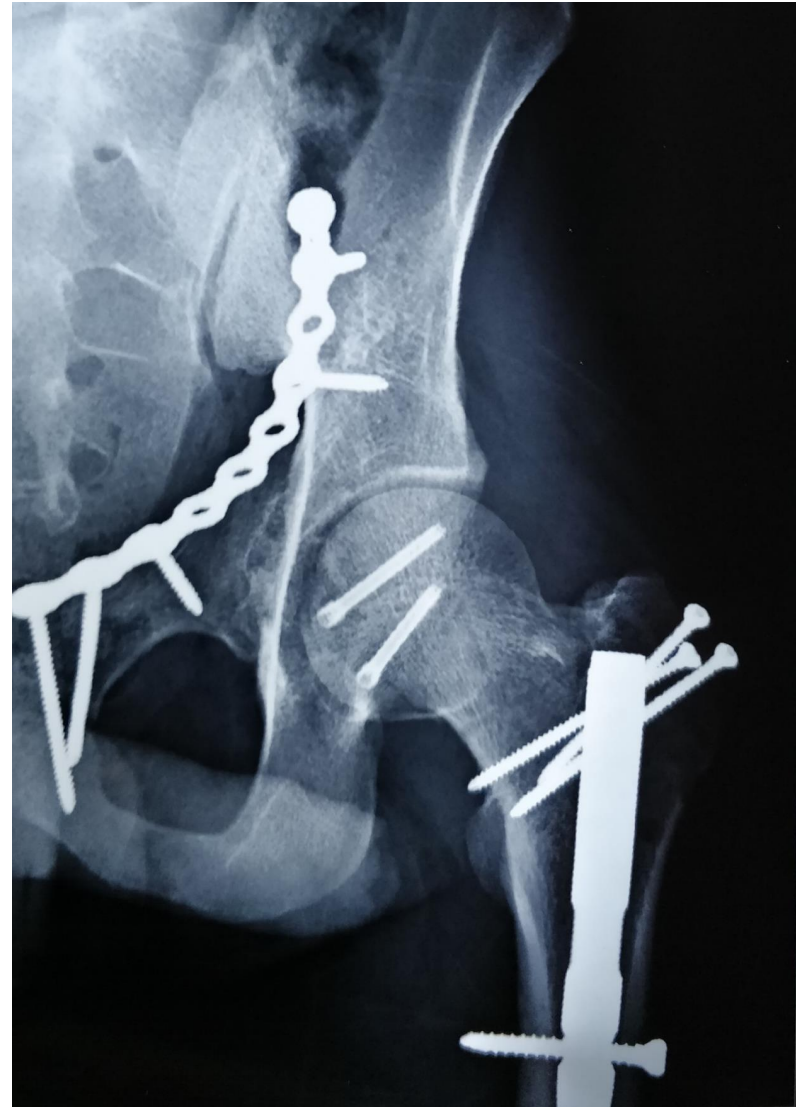

Fig. 2C. - Immediate post-operative radiograph showing fixation of femoral head, femoral shaft and anterior column with headless screws, intramedullary nail, and reconstruction plate, respectively.

3 days by an Ilioinguinal approach (Figures 2A2C). 2 patients with femoral shaft fractures needed a cerclage wiring for stabilization of butterfly fragment during IMN.

Among the 5 patients with femoral neck fractures, 2 had PW fractures. Femoral neck fixation with cannulated screws followed by PW fixation in lateral position in the same sitting was done for them. In the rest, femoral neck fixation was done with cannulated screws on a fracture table. Acetabular fracture fixation was subsequently done after re-draping in the same sitting.

Among the 10 patients with per-trochanteric fractures, proximal femur locking plate fixation (PFLCP) and PW fixation by Kocher Langenbeck (KL) approach were done in lateral position in 4 patients. One patient who received PFLCP was 
operated on by the ilio-inguinal approach in a supine position. Dynamic Hip screw fixation (DHS) was done in 3 patients. Proximal femur nailing (PFN) was done in 2 patients on a fracture table and the acetabular fracture fixation was done in a second sitting. 2 patients had extra-articular distal femur fractures which were managed with a locking compression plate (DFLCP) in the supine position (Tables I and II).

The quality of acetabular reduction based on Matta's criteria on the postoperative radiographs was anatomical in 22 patients, congruent in 10 , and incongruent in 2 patients. Acetabular reduction quality as assessed by chi-square test was not significantly different when the femoral fracture was of the proximal part (femoral neck, head, or pertrochanteric fractures) or the shaft and distal part $(p=0.117)$. In patients with a femoral shaft or distal femur fractures, there were 16 excellent or good reductions and 3 fair or poor reductions. While in those with proximal femur fractures, there were 13 excellent or good reductions and 2 fair or poor reductions. The average postoperative hospital stay was 6.62 days (range, 2-15 days).

Complications were seen in 12 patients. Surgical site infection occurred in 2 patients at the acetabular site. Both were operated in a lateral position in a single sitting using the KL approach for the acetabulum. While one of them improved with intravenous antibiotics, the other required debridement and split skin grafting. One patient with a femoral neck fracture had SSI ; he failed to improve with repeated debridement and required excision of the femoral head. Infected femoral nonunion was seen 6 months after surgery in patient 19 (Tables I and II); he had required cerclage at the time of primary surgery. He was managed with implant removal, debridement, and monorail fixator application. Non-union of the femoral fracture was seen in 3 other patients too. All of them were operated on in a single anaesthetic sitting. 2 patients were managed with exchange nailing and 1 with repeat DFLCP. All of them received autologous iliac crest bone grafts.

In the 2 patients with post-traumatic sciatic nerve palsy, the nerves were intact on exploration. Both improved spontaneously. Iatrogenic sciatic nerve

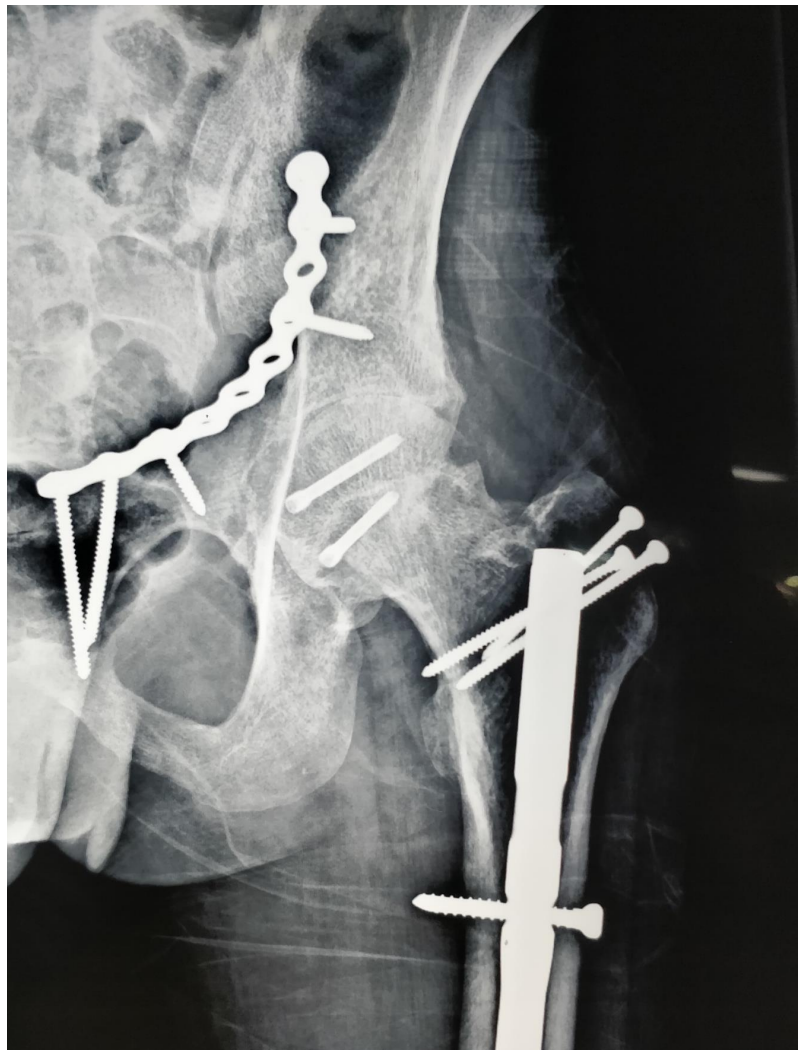

Fig. 2D. - Radiograph at 15 months follow-up showing a reduced hip joint space in patient number 19 (tables I and II). His clinical and radiological outcomes were poor.

palsy occurred in 1 patient, which recovered with supportive treatment. Heterotopic ossification of Brooker grade II was seen in one patient who also had a head injury. Post-operative vascular occlusion occurred in one patient with diaphyseal femur fracture and $\mathrm{T}$ type acetabular fracture. His acetabular fracture was operated in the second sitting by KL followed by IL approaches. He required a bypass procedure by the vascular surgeons. Chondrolysis (Figure 2D) was seen in the patient who had a femoral head fracture (patient 19). Avascular necrosis with secondary arthritis occurred in another patient with a femoral femur neck fracture. The average duration of followup was 30.32 months (range, 12-72 months). The radiological outcome of acetabular fracture was excellent or good in $26(76.47 \%)$ and fair or poor in $8(23.5 \%)$ patients (Table III). However, the clinical outcome was excellent or good in $21(61.7 \%)$ 
Table III. - Table summarizing the details of clinical and radiological outcomes at latest follow up and their comparison relation to the reduction quality assessed on post-operative radiographs.

\begin{tabular}{|c|c|c|c|c|c|}
\hline \multirow{2}{*}{\multicolumn{2}{|c|}{ Outcomes at latest follow-up }} & \multirow{2}{*}{\multicolumn{4}{|c|}{ Reduction quality on post-operative radiographs }} \\
\hline & & & & & \\
\hline \multirow{4}{*}{$\begin{array}{l}\text { Clinical outcome based on } \\
\text { Harris Hip Score (HHS) }\end{array}$} & Excellent & $\begin{array}{l}\text { Anatomical } \\
9 \\
\end{array}$ & $\begin{array}{l}\text { Congruent } \\
0\end{array}$ & $\begin{array}{l}\text { Incongruent } \\
0 \\
\end{array}$ & $\begin{array}{l}\text { 'p' value on Fisher's } \\
\text { exact test }\end{array}$ \\
\hline & Good & 9 & 3 & 0 & \multirow{3}{*}{0.001} \\
\hline & Fair & 1 & 7 & 0 & \\
\hline & Poor & 3 & 0 & 2 & \\
\hline \multirow{4}{*}{$\begin{array}{l}\text { Radiological outcome of } \\
\text { acetabular fracture based } \\
\text { on Matta's score }\end{array}$} & Excellent & 10 & 1 & 0 & \multirow{5}{*}{0.015} \\
\hline & Good & 8 & 7 & 0 & \\
\hline & Fair & 1 & 2 & 0 & \\
\hline & Poor & 3 & 0 & 2 & \\
\hline Total & & 22 & 10 & 2 & \\
\hline
\end{tabular}

patients, fair or poor in $13(38.23 \%)$ patients (Table III). A statistically significant difference was found on Fisher's exact test when the radiological and clinical outcomes were analysed in relation to the post-operative reduction quality ( $\mathrm{p}$ values, 0.015 and 0.001 respectively) (Table III).

\section{DISCUSSION}

Liebergall et al have been credited for coining the term 'floating hip'injuries $(1,5,6)$. Operative treatment of pelvi-acetabular trauma and femur fractures, in isolation is well accepted as the standard of care but literature on floating hip injuries is limited. These injuries occur predominantly in young people and result from road traffic accidents or a fall from significant height. Surgical stabilization helps in rehabilitation and an early return to productivity. While Liebergall et al. (5) preferred operating first on the femur, Siavashi (13) managed 10 out of their 11 patients by operating first on the pelvi-acetabular fractures. Suzuki et al. (3). performed external fixation of pelvic fractures first and then addressed the acetabular and femoral fractures. At our centre, a similar pattern is followed, and the pelvic fracture is addressed first if it has the potential to produce hemodynamic instability. In this series, patients with only concomitant ipsilateral acetabular and femoral fractures (true floating hips injuries) were studied and those with pelvic injuries were excluded.

We noted a male predominance (33:1) and that the patients were primarily young. Liebergall et al. $(1,5)$,
Suzuki et al. (3), and Burd et al. (7) have reported that males were affected more than females in their respective studies. Muller et al. (6) and Siavashi (13) had almost an equal number of patients of each gender. The almost exclusive male involvement in our series can be attributed to their greater outdoor exposure as compared to the females in our region.

Acetabular fractures result from an impact of the femoral head on the acetabulum when forces are transmitted along the femoral shaft to the head, as occurs in dashboard injuries or when forces are transmitted from greater trochanter, as occurs in lateral impact injuries (1,5). Liebergall et al. (5) reported a strong positive correlation between dashboard injury and posterior type of acetabular fractures (PW, PC, T with PW), and between lateral impaction and central type of acetabular fracture (Transverse, T type, BC, AC with PHT or AC with quadrilateral plate fracture). Additionally, they reported a strong correlation between midshaft femoral fractures and posterior type of acetabular fractures and between proximal fractures of the femur and central type of acetabular fractures. In this series, an equal number of patients had a posterior acetabular fracture or an anterior acetabular fracture and either a proximal femur or a femoral shaft fracture ( $\mathrm{n}=7$ and 8 , respectively). 2 patients with distal femur fracture had posterior acetabular fractures. 2 patients had AC and femoral shaft fractures. A distinct correlation between the acetabular and femoral fracture type was not found in the present series. Burd et al. (7) too had failed 
to find any significant correlation between the type of acetabular and femoral fractures. In this series, transverse fracture (10 patients, 29.4\%) was the commonest acetabular fracture type. Muller et al $(36.6 \%)$ and Burd et al $(32.5 \%)$ have reported similar findings $(6,7)$. Sen and Jha (14) in their literature review had concluded that acetabular fractures in floating hip injuries were frequently transverse or posterior wall type or a combination of both. The average time between injury and the first Orthopaedic surgery in this series was 4.41 days and is comparable to that reported by Burd et al (3.6 days) and Muller et al (5.5 days). This resulted primarily from the fact that most patients were referred from other hospitals after initial stabilization.

Femur first strategy has already been reported $(1,3,5)$ and it is postulated to reduce chances of fat embolism (3), facilitate proper positioning, surgical site preparation and draping, exposure, and reduction of the acetabular fractures (7). In the present series, the femoral fractures were always fixed first with the intention that if the surgery could not be completed in one sitting then at least the femur would be stable thereby decreasing the chances of fat embolism and increasing the ease of nursing care. This strategy was reported by Burd et al. (7) too.

In this series, $24(70.6 \%)$ cases were operated on in a single anaesthetic sitting. Burd et al. (7), Liebergall et al. (5) and Muller et al. (6) have operated on $65 \%$, $65 \%$, and $47 \%$ of their patients respectively, in a single sitting. Antegrade femoral nailing can be performed in a lateral position, through an incision which can then be easily incorporated into the KL approach. Even DHS or PFN placement can also be done in lateral position followed by a KL approach for the acetabulum in the same sitting. But in these cases, technical difficulties in fluoroscopic visualization were almost always encountered. Also, an additional scrubbed assistant is frequently needed in these cases. A two-stage surgery was done in 10 (29.4\%) cases, of which 4 cases were of complex acetabular fractures requiring simultaneous or sequential dual approaches. ${ }^{21}$

Quality of reduction has been reported to be an important predictor of clinical and radiological outcomes in acetabular fractures (22). The relationship between quality of reduction and the clinical and radiological outcomes was found to be statistically significant in this study. A comparable analysis has not been performed in previous studies on floating hip injuries. The clinical outcome at recent follow-up was excellent or good in $61.7 \%$ of patients and was inferior when compared to studies that have evaluated the outcomes in isolated acetabular fractures (19,22-24). Zamora-Navas et al ${ }^{18}$ also found inferior results in floating hip cases when compared to isolated acetabular fractures. This might be a result of the additional femoral fracture which can affect a person's mobility and his/her day-to-day activities. Additionally, few patients in this study who had an excellent initial reduction of the acetabular fracture ended up having poor or fair outcomes probably due to the complication seen in them. Infection occurred in 4 patients $(11.76 \%, 2$ on the acetabular side, and 2 on the femoral side). This infection rate was comparable to the $12.1 \%$ rate reported by Zamora-Navas et al. (18) but was higher than the $5-10 \%$ rate reported in isolated acetabular fractures (19,22-24). Acetabular side SSIs were seen in patients operated by the KL approach in the same anaesthetic sitting along with the femoral fracture fixation. Routine prophylaxis with LMWH and Indomethacin was probably helpful as there was only one case of heterotopic ossification and no case of DVT. Iatrogenic sciatic nerve injury occurred in one patient.

There are a few notable shortcomings of this study. The quality of reduction was examined on plain radiographs and CT scans were not obtained out of financial constraints. Scores assessing the severity of trauma were not available as most patients were referred from other centers and invariably had received some initial care. No patient was operated first on the acetabular side thereby introducing an inherent selection bias and hence the ideal sequence of surgery cannot be conclusively stated upon. 16 (around 47\%) patients have a follow-up of fewer than 2 years. The number of patients returning to pre-injury level of activity was not examined.

Inclusion of patients with concomitant acetabular and femoral fractures only and exclusion of pelvic fractures can be considered a strength of this study. The evaluation of outcome measures done by us in relation to the initial reduction quality is an important 
aspect. A detailed description of the complications faced during treatment as presented will be helpful for other surgeons. We believe that this study adds to the existing knowledge on floating hip injuries and would be helpful for decision-making by surgeons planning to manage these complex injuries.

\section{CONCLUSION}

Floating hip injuries with an acetabular component can be managed successfully by a femur first approach with reasonable short-term clinical and radiological outcomes. A randomized study comparing the outcomes of femur first and acetabulum first strategies is warranted to definitively answer the question of which fracture should be operated on first. Complications are not uncommon, and it is imperative for the surgeon to inform the patients about them and the expected relatively poor outcomes when compared to isolated acetabular fractures.

\section{REFERENCES}

1. Liebergall M, Mosheiff R, Safran O, Peyser A, Segal D. The floating hip injury : patterns of injury. Injury. 2002 Oct ; 33(8) : 717-22.

2. Agarwal A, Chadha M. Floating injuries : a review of the literature and proposal for a universal classification. Acta Orthop Belg. 2004 Dec ; 70(6) : 509-14.

3. Suzuki T, Shindo M, Soma K. The floating hip injury : which should we fix first?. European J Orthop Surg Trauma. 2006 Sep 1 ; 16(3) : 214-8.

4. Zamora-Navas P, Guerado E. Vascular complications in floating hip. Hip Int. 2010 ; 20 Suppl 7 : S11-8. Epub 2010 May 27.

5. Liebergall M, Lowe J, Whitelaw GP, Wetzler MJ, Segal D. The floating hip. Ipsilateral pelvic and femoral fractures. J Bone Joint Surg Br. 1992 Jan ; 74(1) : 93-100.

6. Müller EJ, Siebenrock K, Ekkernkamp A, Ganz R, Muhr G. Ipsilateral fractures of the pelvis and the femur-floating hip? A retrospective analysis of 42 cases. Arch Orthop Trauma Surg. 1999 ; 119(3-4) : 179-82.

7. Burd TA, Hughes MS, Anglen JO. The floating hip : complications and outcomes. J Trauma. 2008 Feb ; 64(2) : 442-8.

8. Wu CL, Tseng IC, Huang JW, Yu YH, Su CY, Wu CC. Unstable pelvic fractures associated with femoral shaft fractures : a retrospective analysis. Biomed J. 2013 MarApr ; 36(2) : 77-83.
9. Wiltberger BR, Mitchell CL, Hedrick DW. Fracture of the femoral shaft complicated by hip dislocation - a method of treatment. J Bone Joint Surg Am. 1948 Jan ; 30A(1) : 2258.

10. Wu CC, Shih CH, Chen LH. Femoral shaft fractures complicated by fracture-dislocations of the ipsilateral hip. J Trauma. 1993 Jan ; 34(1) : 70-5.

11. Helal B, Skevis X. Unrecognised dislocation of the hip in fractures of the femoral shaft. J Bone Joint Surg Br. 1967 May ; 49(2) : 293-300.

12. Iotov A, Tzachev N, Enchev D, Baltov A. Operative treatment of the floating hip. In Orthopaedic Proceedings 2006 Mar (Vol. 88, No. Supp. I, pp. 160-160). The British Editorial Society of Bone \& Joint Surgery.

13. Siavashi B. Floating Hip, Eleven Cases and Literature Review. Journal of Orthopedic and Spine Trauma. 2017 March ; 3(1) : e63868. doi : 10.5812/jost.63868.

14. Sen RK, Jha L. Floating hip. J Clin Orthop. 2017 Jan ; 2(1) : 43-8.

15. Rajasekaran RB, Jayaramaraju D, Palanisami DR, Perumal R, Shanmuganathan R. Ipsilateral Acetabular Fracture with Displaced Femoral Head and Femoral Shaft Fracture : A Complex Floating Hip Injury. Case Rep Orthop. 2018 Jul 3 ; 2018 : 4937472.

16. Tiedeken NC, Saldanha V, Handal J, Raphael J. The irreducible floating hip : a unique presentation of a rare injury. J Surg Case Rep. 2013 Oct 4 ; 2013(10).

17. Irifune H, Hirayama $S$, Takahashi N, Narimatsu E. Ipsilateral Acetabular and Femoral Neck and Shaft Fractures. Case Rep Orthop. 2015 ; 2015 : 351465.

18. Zamora-Navas P, Estades-Rubio FJ, Cano JR, Guerado E. Floating hip and associated injuries. Injury. $2017 \mathrm{Nov}$; 48 Suppl 6 : S75-S80.

19. Matta JM. Fractures of the acetabulum : accuracy of reduction and clinical results in patients managed operatively within three weeks after the injury. J Bone Joint Surg Am. 1996 Nov ; 78(11) : 1632-45.

20. Matta JM, Merritt PO. Displaced acetabular fractures. Clin Orthop Relat Res. 1988 May ; (230) : 83-97.

21. Meena UK, Chand Bansal M, Singh J, Behera $P$, Kulkarni C. Can patients with complex acetabular fractures be operated by combined anterior and posterior approaches in a single anesthetic sitting? J Orthop Sci. 2020 Nov ; 25(6) : 1021-1028.

22. Meena UK, Tripathy SK, Sen RK, Aggarwal S, Behera P. Predictors of postoperative outcome for acetabular fractures. Orthop Traumatol Surg Res. 2013 Dec ; 99(8) : 929-35.

23. Briffa N, Pearce R, Hill AM, Bircher M. Outcomes of acetabular fracture fixation with ten years' follow-up. $J$ Bone Joint Surg Br. 2011 Feb ; 93(2) : 229-36.

24. Madhu R, Kontis A, Mousawi A. Outcome of surgery for reconstruction of fractures of acetabulum: the time dependent effect of delay. J Bone Joint Surg Br 2006 ; 36 : 451-75. 